Vol. 6, No. 2, 2020

Vitaliy Korendiy ${ }^{1}$, Oleksandr Kachur ${ }^{2}$, Oleksandr Havrylchenko ${ }^{3}$, Vasyl Lozynskyy ${ }^{4}$

${ }^{1}$ Department of Mechanics and Automation Engineering, Lviv Polytechnic National University, 12, S. Bandery Str., Lviv, Ukraine, e-mail: vitaliy.nulp@gmail.com, ORCID 0000-0002-6025-3013

${ }^{2}$ Department of Mechanics and Automation Engineering, Lviv Polytechnic National University, 12, S. Bandery Str., Lviv, Ukraine, e-mail: dingof1992@gmail.com, ORCID 0000-0003-2263-6360

${ }^{3}$ Department of Mechanics and Automation Engineering, Lviv Polytechnic National University, 12, S. Bandery Str., Lviv, Ukraine, e-mail: gavr40644@gmail.com, ORCID 0000-0002-9596-3322

${ }^{4}$ Department of Electronics and Information Technology, Lviv Polytechnic National University, 12, S. Bandery Str., Lviv, Ukraine, e-mail: Vasyl.I.Lozynskyi@lpnu.ua, ORCID 0000-0001-8948-239X

\title{
MODELLING AND SIMULATION OF PNEUMATIC SYSTEM OPERATION OF MOBILE ROBOT
}

Received: March 14, 2020 / Revised: July 28, 2020 / Accepted: September 30, 2020

(C) Korendiy V., Kachur O., Havrylchenko O., Lozynskyy V., 2020

Abstract. Problem statement. Mobile robots are currently of significant interest among researchers and designers all over the world. One of the prospective drives of such robots is equipped by a pneumatically operated orthogonal system. The processes of development and improvement of orthogonal walking robots are significantly constrained because of the lack of an open-access comprehensive scientific and theoretical framework for calculating and designing of the energy-efficient and environmental-friendly pneumatic walking drives. Purpose. The main purpose of this research consists in the kinematic analysis, motion modelling and pneumatic system simulation of the mobile robot with an orthogonal walking drive. Methodology. The research is carried out using the basic laws and principles of mechanics, pneumatics and automation. The numerical modelling of the robot motion is conducted in MathCad software. The computer simulation of the robot kinematics is performed using SolidWorks software. The operational characteristics of the robot's pneumatic system are investigated in Festo FluidSim software. Findings (results) and originality (novelty). The improved design of the mobile robot equipped by the orthogonal walking drive and turning mechanism is thoroughly investigated. The motion equations of the orthogonal walking drive are deduced, and the graphical dependencies describing the trajectories (paths) of the robot's feet and body are constructed. The pneumatically operated system ensuring the robot rectilinear and curvilinear locomotion is substantiated. Practical value. The proposed design of the walking robot can be used while developing industrial (production) prototypes of mobile robotic systems intended for performing various activities in the environments that are not suitable for using electric power. Scopes of further investigations. While carrying out further investigations, it is expedient to design the devices for changing the robot locomotion speed and controlling the lifting height of its feet.

Keywords: pneumatic drive, walking robot, orthogonal system, kinematic analysis, turning mechanism, curvilinear locomotion, trajectory.

\section{Introduction}

The walking (stepping) method is primarily used to enable the locomotion of various transport and technological machines in the arbitrary natural conditions and on a rough terrain. This is especially the case when the use of wheeled or tracked drives is inexpedient or impossible. The walking machines can be effectively used for military purposes, scientific research, in forestry, horticulture and agriculture. The involvement of the walking (stepping) machines is quite expedient during the introduction of prospective 


\section{Vitaliy Korendiy, Oleksandr Kachur, Oleksandr Havrylchenko, Vasyl Lozynskyy}

soil-saving and environmental-friendly technologies in agriculture, because the walking (stepping) method of locomotion provides a discrete impact of supporting elements on the ecologically vulnerable soil surface, while the wheeled or tracked vehicles create continuous tracks destroying the fertile soil layers.

\section{Problem Statement}

The locomotion of large and heavy transport and technological machines involves the need to ensure the stability of the machine's body position while moving, the specified locomotion speed, manoeuvrability and passability. The first two tasks are vey important because of the significant power losses that take place during periodic lifting-lowering and accelerating-decelerating of the machine's body while walking. These losses can be controlled by the kinematics of the walking (stepping) mechanisms used in the drives of the supporting elements. On the other hand, in the case of increasing the machine mass, the problems of eliminating the dynamic loads exerted upon the elements of the walking drive while performing the locomotion become more and more urgent. Therefore, the problems of modelling (simulation) and analysis of the motion conditions of the walking machines, as well as the substantiation of design parameters and operational characteristics of their drives are currently relevant.

\section{Review of Modern Information Sources on the Subject of the Paper}

Mobile robotic systems are currently of significant interest among researchers and designers all over the world. The numerous scientific and educational publications are widely available. The general information about the most widespread designs and areas of implementation of mobile robots is presented in [1]. Basic theories, methods and algorithms used for studying mobile robotic systems are considered in [2]. The problems dealing with the dynamics and control of robotic systems are analyzed in [3]. Most of existent educational publications are dedicated to the robotic systems equipped by a wheeled or a caterpillar drive, whereas the problems of development of the walking robots are not thoroughly analyzed.

The paper [4] is dedicated to the kinematic analysis of the orthogonal robot with four degrees of freedom. In [5], there are considered the algorithms of controlling the orthogonal robot motion depending on the signals obtained from the spatial orientation system. The problems of synthesizing the optimal laws of the orthogonal robot locomotion aimed at reducing the power consumption are investigated in [6]. The investigations dedicated to the implemented methodologies of performing the in-pipe locomotion of various mobile robots driven by different actuators (DC motors, servo motors, pneumatic and hydraulic systems) are presented in [7]. Most of the considered publications deal with mobile robots that are equipped with electric drives while the problems of development of the pneumatically driven orthogonal walking (stepping) robots are not thoroughly investigated.

This paper continues the authors' research on the operation of mobile robots with pneumaticallydriven walking mechanisms. These investigations have been initiated in [8], where the general design of the robot was proposed and its operational characteristics were considered. The paper [9] extends the obtained results to modelling and simulation of the robot motion. In [10], there was performed the structural and kinematic analysis of the pantograph-type manipulator with three degrees of freedom used for conducting the prescribed technological operations. The paper [11] was dedicated to improvement of the design parameters of the pneumatically-driven orthogonal robot able to perform rectilinear locomotion (Fig. 1). All the considered papers became the basis for the current research dealing with the curvilinear locomotion of the developed mobile robotic system additionally equipped with the turning mechanism.

\section{Objectives and Problems of Research}

The main purpose of the paper consists in development of the improved design of the pneumaticallydriven orthogonal walking robot able to perform both rectilinear and curvilinear locomotion. The major tasks set in this research are following: constructing the improved pneumatic system of the robot's drive; deriving the mathematical expressions describing the laws of motion of the robot's members; performing the numerical modelling of the robot locomotion in MathCad software; simulation of the robot locomotion in SolidWorks software; simulating the operation of the robot's pneumatic system in Festo FluidSim software. 


\section{Modelling and Simulation of Pneumatic System Operation of Mobile Robot}

\section{Pneumatic Circuit of the Robot's Drive}

The general design (Fig. 1) and operational peculiarities of the four-degree-of-freedom doublelegged robot with pneumatic drive and turning mechanism are thoroughly analyzed in [11]. In particular, the previous authors' paper [11] presents the improved design of the pneumatically-driven orthogonal walking robot equipped with turning mechanism, analyses its rectilinear and curvilinear locomotion, considers the corresponding timing diagrams of operation of the pneumatic cylinders under the conditions of rectilinear and curvilinear motion. The present paper continues the investigations initiated in [11].

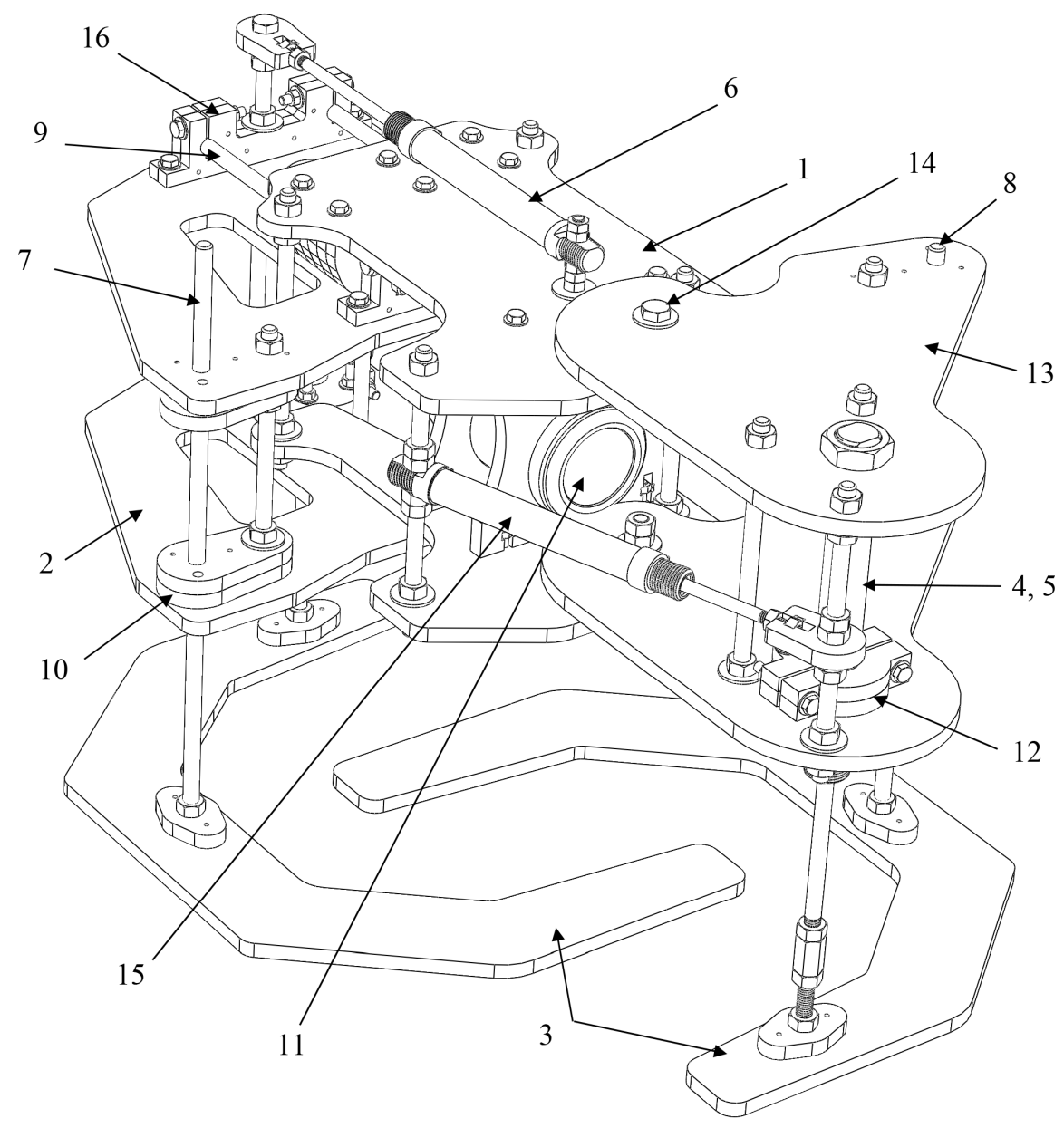

Fig. 1. Improved design of laboratory-scale mobile robot: 1, 2 - movable platforms; 3 - feet;

4, 5, 6, 15 - pneumatic cylinders; 7, 8, 9- guides (guiding rods); 10 - linear bearings; 11 - air receiver;

12 - pneumatic cylinder fixator; 13 - turning platform; 14 - cylindrical hinge; 16 - guide fixator

The pneumatic circuit describing the control system of operation of the robot's pneumatic cylinders during its curvilinear motion has been developed in [11] (Fig. $2 a$ ). The principal pneumatic diagram consists of four double-acting (two-way) cylinders providing direct and reverse run (Д1, Д2, Д3, Д4) [12]; four two-position four-line pneumatic control distribution valves $(P 1, P 4, P 7, P 11)$, six two-position four-line pneumatic distribution valves with end-sensors $(P 2, P 3, P 5, P 6, P 8, P 9)$, two logical adding (summing) elements $(I 1, I 2)$, starting distribution valve $P 10$ ("Start" button for starting the rectilinear motion), starting distribution valve $P 12$ ("Start-2" button for starting the turning process), and eight regulating (pilot) airflow constrictors with check (non-return) valves for providing the possibility of changing the intensity of air-consumption to the corresponding cylinders (i.e., for changing the motion speeds of their piston-rods).

After pushing the "Start" button, while the "Start-2" button is not pushed, the compressed air is supplied from the pneumatic system and switches the control distribution valve $P 11$, as a result of which the rod of the pneumatic cylinder $Д 4$, which is responsible for turning the robot, is fixed in the extended 


\section{Vitaliy Korendiy, Oleksandr Kachur, Oleksandr Havrylchenko, Vasyl Lozynskyy}

position. This position of a rod of the turning pneumatic cylinder provides rectilinear motion of the robot. That is, when the "Start-2" button is not pushed, there takes place the stepping cyclogram (timing diagram) shown in Fig. 2 b. After pushing the "Start" button, the air flows through the ports (passages) of the distribution valve $P 10$ if the piston of the cylinder $\not 3$ is located in the left end position. The air flows from the air-supply system to the control distribution valves $P 4, P 7$ providing the reverse run (back stroke) of the rods of the pneumatic cylinders $Д 2$ and the direct run (forward stroke) of the rod of the cylinder Д3. Taking into account the necessity to ensure the simultaneous operation of two pneumatic cylinders during the first cycle, one logical adding (summing) element $I 2$ was used for registering the positions of the corresponding piston-rods with tappets of the pneumatic cylinders $Д 2$ and $Д 3$ during the stroke 1 . The output signal from the adding (summing) element $I 2$ switches the control distribution valves $P 1$ and $P 4$. This provides the direct run (forward stroke) of the piston of the cylinder $Д 2$ and the reverse run (back stroke) of the piston of the cylinder Д1.

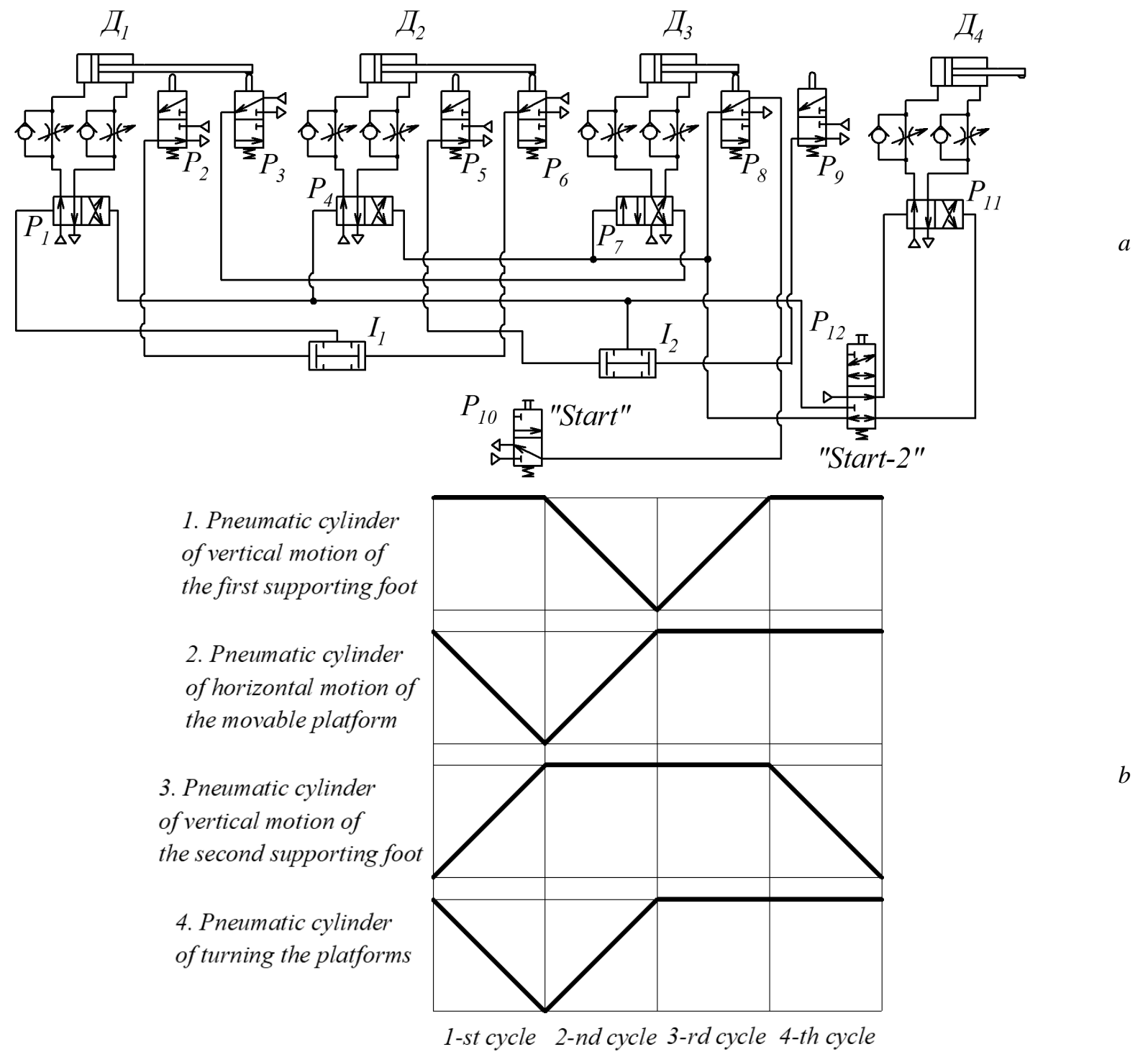

Fig. 2. Pneumatic circuit of the robot's drive $(a)$ and cyclogram (timing diagram) of its curvilinear motion $(b)$

In order to start the stroke 3 , it is necessary to register the end of performing the stroke 2 . That is why the logical adding (summing) element $I_{1}$ is used in the pneumatic circuit. This element registers the end of the stroke 2 (the end positions of the piston-rods with tappets of the pneumatic cylinders $Д_{1}$ and $Д_{2}$ ) due to adding (summing) the signals of the distribution valves with end-sensors $P_{2}$ and $P_{6}$. The logical adding (summing) element $I_{1}$ gives a command for performing the stroke 3 (the direct run (forward stroke) 
of the piston of the cylinder $Д_{1}$ ) by means of changing the position of the control distribution valve $P_{1}$. When the piston-rod of the cylinder $Д_{1}$ reaches its end-position, the distribution valve $P_{3}$ gives a command for performing the reverse run (back stroke) of the rod of the cylinder $Д_{3}$ by switching the distribution valve $P_{7}$. At the end of the fourth stroke, the full cycle of the pneumatic system operation is performed. After this the following walking cycle is to be performed.

In order to stop the operation of the pneumatic system (and the cyclic stepping process of the robot) it is necessary to push the "Start" button (the starting the distribution valve $P_{10}$ ).

When the "Start-2" button (starting distribution valve P12) is switched on (pushed), the compressed air is supplied to the control distribution valve $P 11$, which controls the operation of the turning pneumatic cylinder Д4. At the same time, the control distribution valve $P 4$ responsible for operation of the pneumatic cylinder $Д 2$ is supplied by the compressed air according to the same cyclogram as for the control distributor $P 11$. The pneumatic cylinder $Д 2$ sets into horizontal motion the movable platforms. Thus, due to the use of a two-position five-line starting distribution valve $P 12$ it is possible to synchronize the operation of the pneumatic cylinders Д2 and Д4, and to implement the specified process of the robot stepping with the possibility of changing the direction of its course motion (turning).

\section{Simulating the Operation of the Robot's Pneumatic System in Festo FluidSim Software}

In order to substantiate the adequacy and operation capacity of the proposed principal pneumatic circuit of the mobile robot (Fig. 2), the corresponding simulation model of the pneumatic system was implemented in applied software FESTO FluidSim Pneumatics (Fig. 3). The results of simulation presented in the form of timing diagrams of the pneumatic cylinders operation are shown in Fig. 3. Analysing the obtained results, we can state that the timing diagram obtained by simulation in FESTO FluidSim Pneumatics software satisfactorily corresponds to the theoretical timing diagram (Fig. 2).

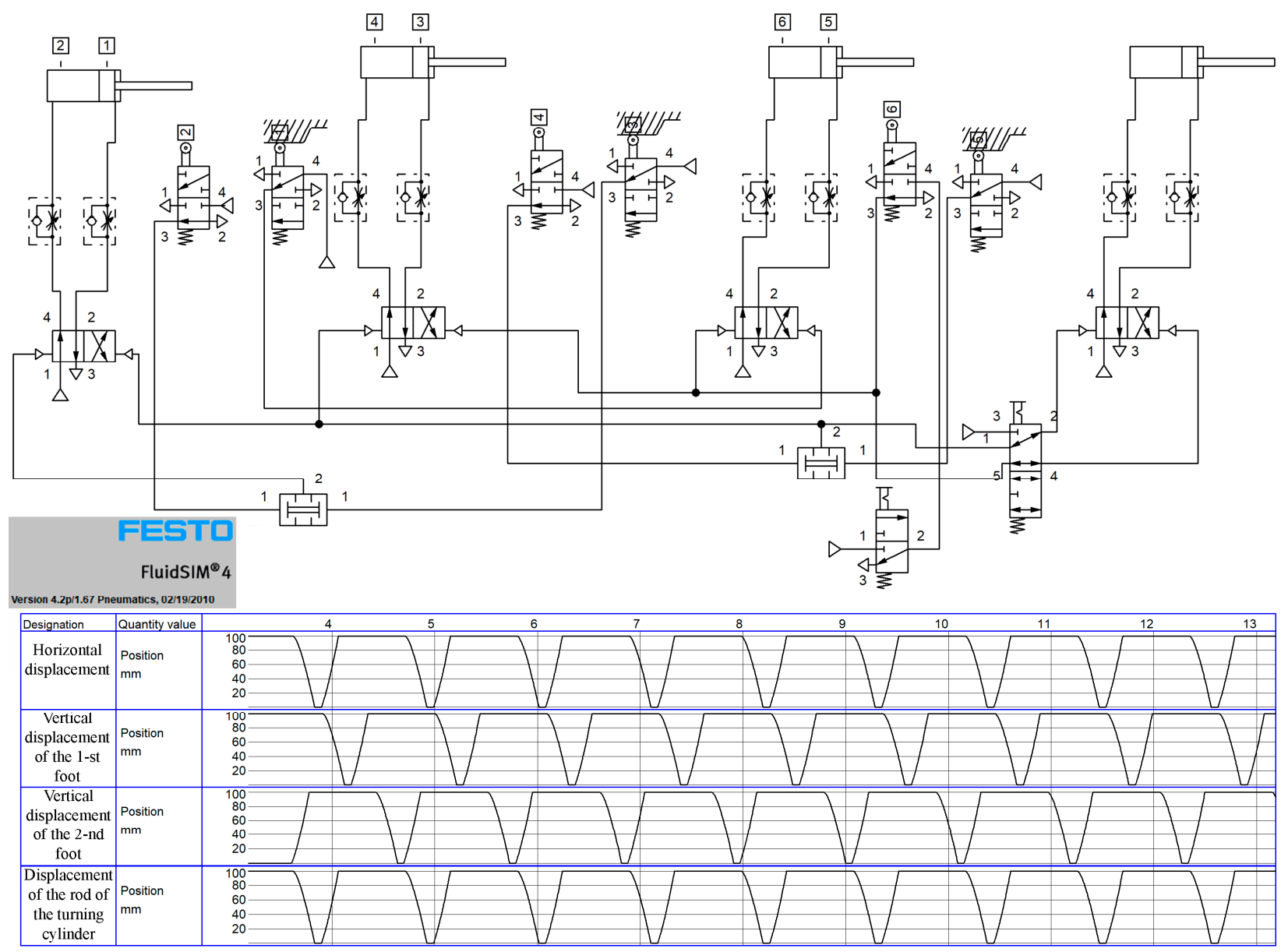

Fig. 3. Results of simulation performed in FESTO FluidSim Pneumatics software 


\section{Vitaliy Korendiy, Oleksandr Kachur, Oleksandr Havrylchenko, Vasyl Lozynskyy}

\section{Kinematic Diagram of the Robot's Mechanical System}

In order to perform further modelling of the robot motion, let us construct the kinematic diagram of its mechanical system (Fig. 4). The supporting feet 1 and 2 can move along the guides $B$ and $C$, respectively. The guides are modelled as prismatic sliders allowing the translational motion and restricting the rotation of the guiding rods. The guides are attached to the corresponding movable platforms 3 and 4 . The generalized coordinates describing the vertical motion of the supporting feet are denoted as variables $y_{1}$ and $y_{2}$, which express the laws of motion of the rods of the corresponding vertical pneumatic cylinders (Fig. 1). The platforms 3 and 4 can translationally move with respect to each other along the horizontal guide $O$, which is modelled as a prismatic slider attached to one of the movable platforms. The generalized coordinate describing the relative translational motion of the platforms is denoted as $x_{1}$, which expresses the law of motion of the rod of the corresponding horizontal pneumatic cylinder (Fig. 1). The cylindrical hinge $A$ allows the turning of the movable platforms 3 and 4 with respect to each other. The generalized coordinate describing the relative angular position of the platforms is denoted as $\varphi_{1}$.

The walking mechanism is characterized by four degrees of freedom, which can be clearly described by the corresponding generalized $x_{1}, y_{1}, y_{2}$ and $\varphi_{1}$. To perform further kinematic analysis let us adopt the Cartesian (rectangular) coordinate system $O x y z$ with its center (origin) located at slider $O$ (Fig. 4).

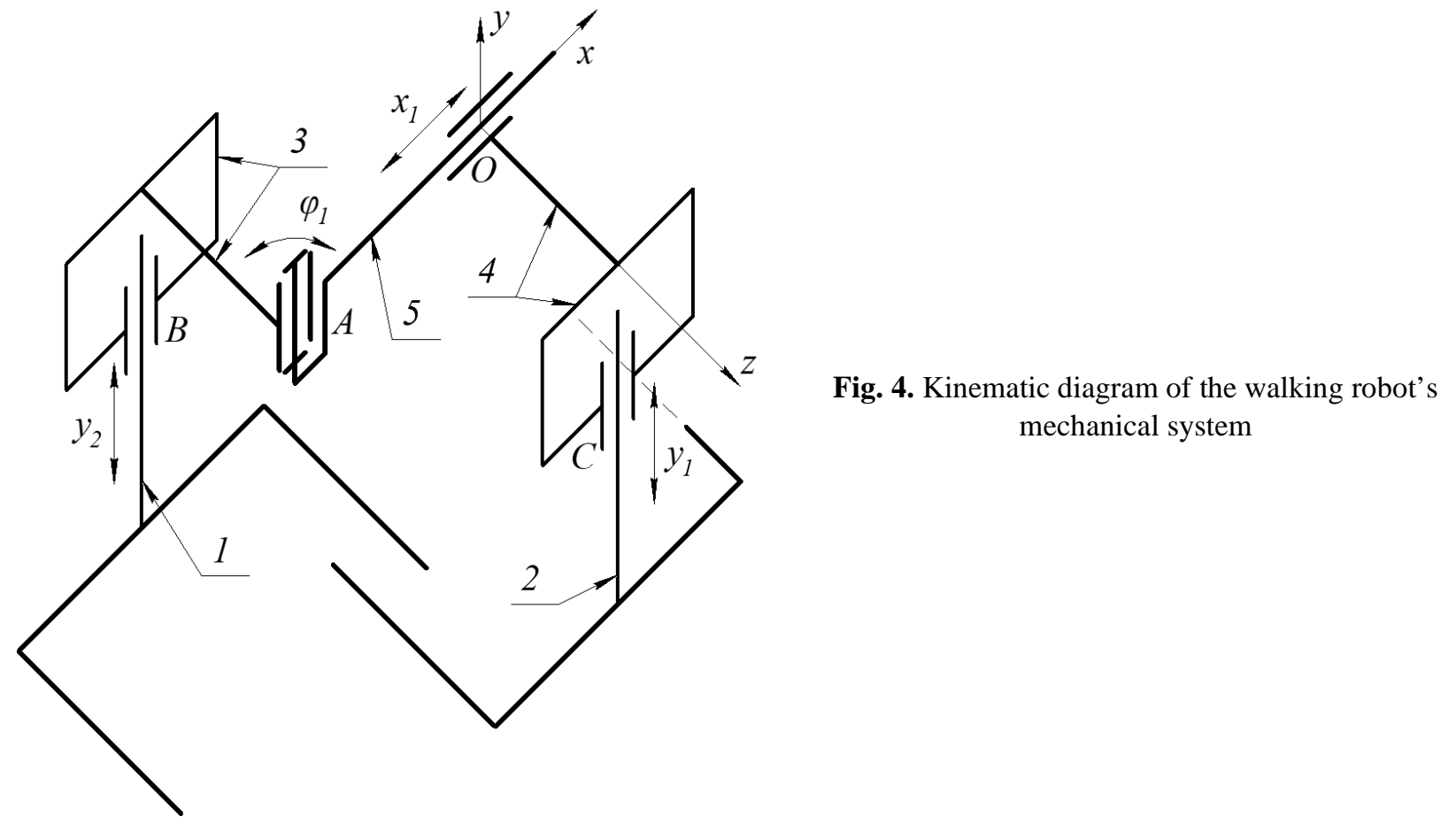

Laws of Motion of the Robot's Members

On the basis of the constructed timing diagram of the mobile robot walking process (Fig. 2), let us derive the equations of motion of the robot's members. Let us make the following assumptions: 1) the duration $T$ of each cycle (stroke) of the robot's walking process is equal (unchanged); 2) the speed $V$ of motion of each piston-rod of all pneumatic cylinders in the forward and back stroke (direct and reverse run) is equal (for the pneumatic cylinders of the platforms' horizontal motion $V_{h}=\dot{x}_{1}=\dot{x}_{2}$; for the pneumatic cylinders of the feet vertical motion $\left.V_{v}=\dot{y}_{1}=\dot{y}_{2}\right)$; 3) the speed of motion of the rod of each pneumatic cylinder during one stroke is constant $\left(V_{h}=\right.$ const,$V_{v}=$ const $\left.) ; 4\right)$ the angular speed of turning of one movable platform with respect to another one during one stroke is constant ( $\omega=\dot{\varphi}_{1}=$ const $)$.

Taking into account the assumptions made, in accordance with the proposed timing diagram of the robot's pneumatic system operation (Fig. 2), let us derive the laws of motion of the load-carrying frame 


\section{Modelling and Simulation of Pneumatic System Operation of Mobile Robot}

(body) and of the supporting feet during one step with simultaneous turning of the robot's platforms. Let us assume that $x_{1}$ is the horizontal displacement of the movable platforms with respect to one another; $y_{1}$, $y_{2}$ are the related vertical displacements of the supporting feet with respect to the corresponding movable platforms; $\varphi_{1}$ is the angle of turning of one movable platform with respect to another one). The equations describing the robot's members motion are following:

$$
\begin{aligned}
& x_{\text {foot } 2}(t)=\left\{\begin{array}{l}
0,0 \leq t \leq T ; \\
\dot{x}_{1} \cdot(t-T) \cdot \cos \left(\dot{\varphi}_{1} \cdot(t-T)\right)-O C \cdot \sin \left(\dot{\varphi}_{1} \cdot(t-T)\right), T \leq t \leq 2 \cdot T ; \\
\dot{x}_{1} \cdot T \cdot \cos \left(\dot{\varphi}_{1} \cdot T\right)-O C \cdot \sin \left(\dot{\varphi}_{1} \cdot T\right), 2 \cdot T \leq t \leq 3 \cdot T ; \\
\dot{x}_{1} \cdot T \cdot \cos \left(\dot{\varphi}_{1} \cdot T\right)-O C \cdot \sin \left(\dot{\varphi}_{1} \cdot T\right), 3 \cdot T \leq t \leq 4 \cdot T .
\end{array}=\right. \\
& =\left\{\begin{array}{l}
0,0 \leq t \leq T \\
V_{h} \cdot(t-T) \cdot \cos (\omega \cdot(t-T))-O C \cdot \sin (\omega \cdot(t-T)), T \leq t \leq 2 \cdot T \\
V_{h} \cdot T \cdot \cos (\omega \cdot T)-O C \cdot \sin (\omega \cdot T), 2 \cdot T \leq t \leq 3 \cdot T \\
V_{h} \cdot T \cdot \cos (\omega \cdot T)-O C \cdot \sin (\omega \cdot T), 3 \cdot T \leq t \leq 4 \cdot T
\end{array}\right. \\
& x_{\text {foot } 1}(t)=\left\{\begin{array}{l}
\dot{x}_{1} \cdot t, 0 \leq t \leq T ; \\
\dot{x}_{1} \cdot T, T \leq t \leq 2 \cdot T ; \\
\dot{x}_{1} \cdot T, 2 \cdot T \leq t \leq 3 \cdot T ; \\
\dot{x}_{1} \cdot T, 3 \cdot T \leq t \leq 4 \cdot T .
\end{array}=\left\{\begin{array}{l}
V_{h} \cdot t, 0 \leq t \leq T ; \\
V_{h} \cdot T, T \leq t \leq 2 \cdot T ; \\
V_{h} \cdot T, 2 \cdot T \leq t \leq 3 \cdot T ; \\
V_{h} \cdot T, 3 \cdot T \leq t \leq 4 \cdot T .
\end{array}\right.\right. \\
& y_{\text {foot } 2}(t)=\left\{\begin{array}{l}
-y_{1 \min }, 0 \leq t \leq T ; \\
-y_{1 \min }+\dot{y}_{1} \cdot(t-T), T \leq t \leq 2 \cdot T ; \\
-y_{1 \min }+\dot{y}_{1} \cdot T-\dot{y}_{1} \cdot(t-2 \cdot T), 2 \cdot T \leq t \leq 3 \cdot T ; \\
-y_{1 \min }, 3 \cdot T \leq t \leq 4 \cdot T .
\end{array}=\left\{\begin{array}{l}
-y_{1 \min }, 0 \leq t \leq T ; \\
-y_{1 \min }+V_{v} \cdot(t-T), T \leq t \leq 2 \cdot T ; \\
-y_{1 \min }+V_{v} \cdot T-V_{v} \cdot(t-2 \cdot T), 2 \cdot T \leq t \leq 3 \cdot T ; \\
-y_{1 \min }, 3 \cdot T \leq t \leq 4 \cdot T .
\end{array}\right.\right. \\
& y_{\text {foot } 1}(t)=\left\{\begin{array}{l}
-y_{2 \min }+\dot{y}_{1} \cdot T-\dot{y}_{1} \cdot t, 0 \leq t \leq T ; \\
-y_{2 \min }, T \leq t \leq 2 \cdot T ; \\
-y_{2 \min }, 2 \cdot T \leq t \leq 3 \cdot T ; \\
-y_{2 \min }+\dot{y}_{2} \cdot(t-3 \cdot T), 3 \cdot T \leq t \leq 4 \cdot T .
\end{array}=\left\{\begin{array}{l}
-y_{2 \min }+V_{v} \cdot T-V_{v} \cdot t, 0 \leq t \leq T ; \\
-y_{2 \min }, T \leq t \leq 2 \cdot T ; \\
-y_{2 \min }, 2 \cdot T \leq t \leq 3 \cdot T ; \\
-y_{2 \min }+V_{v} \cdot(t-3 \cdot T), 3 \cdot T \leq t \leq 4 \cdot T .
\end{array}\right.\right. \\
& z_{\text {foot } 2}(t)=\left\{\begin{array}{l}
O C, 0 \leq t \leq T ; \\
\dot{x}_{1} \cdot(t-T) \cdot \sin \left(\dot{\varphi}_{1} \cdot(t-T)\right)+O C \cdot \cos \left(\dot{\varphi}_{1} \cdot(t-T)\right), T \leq t \leq 2 \cdot T ; \\
\dot{x}_{1} \cdot T \cdot \sin \left(\dot{\varphi}_{1} \cdot T\right)+O C \cdot \cos \left(\dot{\varphi}_{1} \cdot T\right), 2 \cdot T \leq t \leq 3 \cdot T ; \\
\dot{x}_{1} \cdot T \cdot \sin \left(\dot{\varphi}_{1} \cdot T\right)+O C \cdot \cos \left(\dot{\varphi}_{1} \cdot T\right), 3 \cdot T \leq t \leq 4 \cdot T .
\end{array}=\right. \\
& =\left\{\begin{array}{l}
O C, 0 \leq t \leq T \\
V_{h} \cdot(t-T) \cdot \sin (\omega \cdot(t-T))+O C \cdot \cos (\omega \cdot(t-T)), T \leq t \leq 2 \cdot T \\
V_{h} \cdot T \cdot \sin (\omega \cdot T)+O C \cdot \cos (\omega \cdot T), 2 \cdot T \leq t \leq 3 \cdot T \\
V_{h} \cdot T \cdot \sin (\omega \cdot T)+O C \cdot \cos (\omega \cdot T), 3 \cdot T \leq t \leq 4 \cdot T .
\end{array}\right. \\
& z_{\text {cmonu } 1}(t)=\left\{\begin{array}{l}
-A B, 0 \leq t \leq T \\
-A B, T \leq t \leq 2 \cdot T \\
-A B, 2 \cdot T \leq t \leq 3 \cdot T \\
-A B, 3 \cdot T \leq t \leq 4 \cdot T
\end{array}\right.
\end{aligned}
$$

where $y_{1 \min }, y_{2 \min }$ are the maximal extensions of the rods of vertical pneumatic cylinders of the supporting feet (i.e. the lowest positions of the supporting feet on the vertical axis); $x_{\text {foot } 1}, x_{\text {foot } 2}, y_{\text {foot } 1}$, 
Vitaliy Korendiy, Oleksandr Kachur, Oleksandr Havrylchenko, Vasyl Lozynskyy

$y_{\text {foot } 2}, z_{\text {foot } 1}, z_{\text {foot } 2}$ are the coordinates of the corresponding members of the robot's mechanical system; $O C$ is the smallest distance between the axes of the hinges $O$ and $C ; A B$ is the smallest distance between the axes of the hinges $A$ and $B$.

\section{Numerical Modelling of the Robot Rectilinear Locomotion in MathCad Software}

Using the derived equations (1) of motion of the considered mobile robot, let us simulate the displacements of its members during its rectilinear locomotion $\left(\dot{\varphi}_{1}=\omega=0\right)$ in MathCAD software. Firstly, let us specify the input parameters of modelling: 1) nominal speed of the piston-rods of the pneumatic cylinders of horizontal motion of the movable platforms and of vertical motion of the supporting feet $V_{h}=V_{v}=0.08 \mathrm{~m} / \mathrm{s} ; 2$ ) duration of one walking cycle (stroke) $T=2 \mathrm{~s} ; 3$ ) maximal extension of the pistonrods of the vertical and horizontal pneumatic cylinders $y_{1 \min }=y_{2 \min }=x_{1 \max }=0.08 \mathrm{~m}$.

Taking into account the specified above parameters of the pneumatic system of the mobile robot, the time dependencies of vertical and horizontal coordinates of the supporting feet of the walking mechanism and the motion trajectories (paths) of the supporting feet are presented in Fig. 5 for the first three steps.
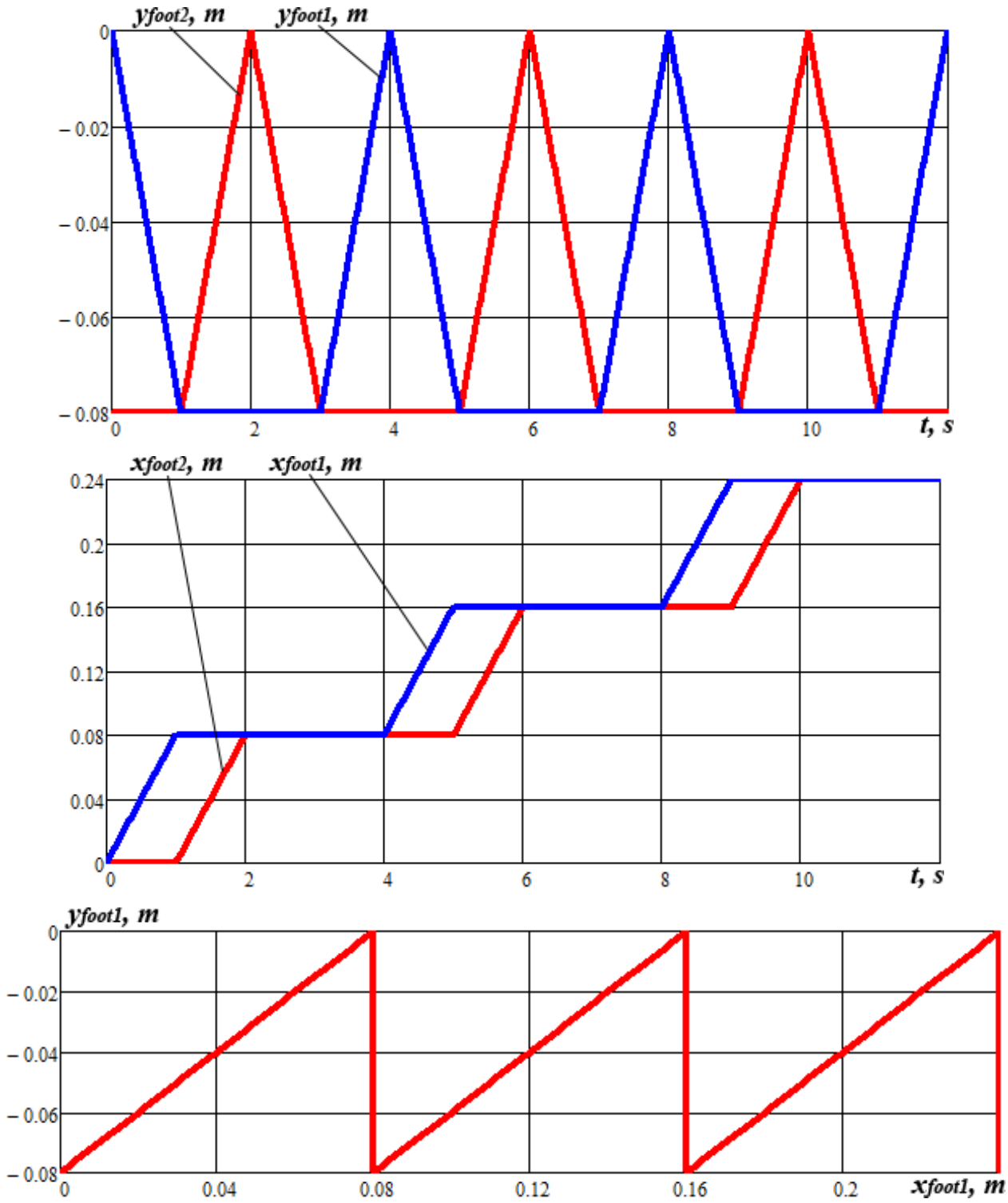

Fig. 5. Time dependencies of changing the coordinates of the robot's supporting feet $(a, b)$ and the motion trajectory (path) of the supporting foot $(c)$ 


\section{Modelling and Simulation of Pneumatic System Operation of Mobile Robot}

\section{Numerical Modelling of the Robot Turning Process}

Let us adopt the same input parameters prescribed above. The maximal angle of turning one platform with respect to another one is limited by the robot's design parameters and is equal $\varphi_{\max } \approx 30^{\circ}$. This turn must be performed during the first walking cycle, i.e. during the time period $T=1 \mathrm{~s}$. The angular velocity of the turning process is considered to be constant and equal to:

$$
\omega=\frac{\pi \cdot \varphi_{\max }}{T \cdot 180^{\circ}}=\frac{\pi \cdot 30^{\circ}}{1 \cdot 180^{\circ}}=0.523 \mathrm{rad} / \mathrm{s} .
$$

Prescribing the length $O C=0.1 \mathrm{~m}$ (Fig. 4), based on the equations (1) let us simulate the robot turning process during the first three steps. The results of simulation are presented in the form of time dependencies of the coordinates of the robot's foot center during the first $12 \mathrm{~s}$ (Fig. $6 \mathrm{a}$ ), and of the trajectory of the foot indicating the specific points of the foot contact with the supporting surface (Fig. $6 \mathrm{~b}$ ).
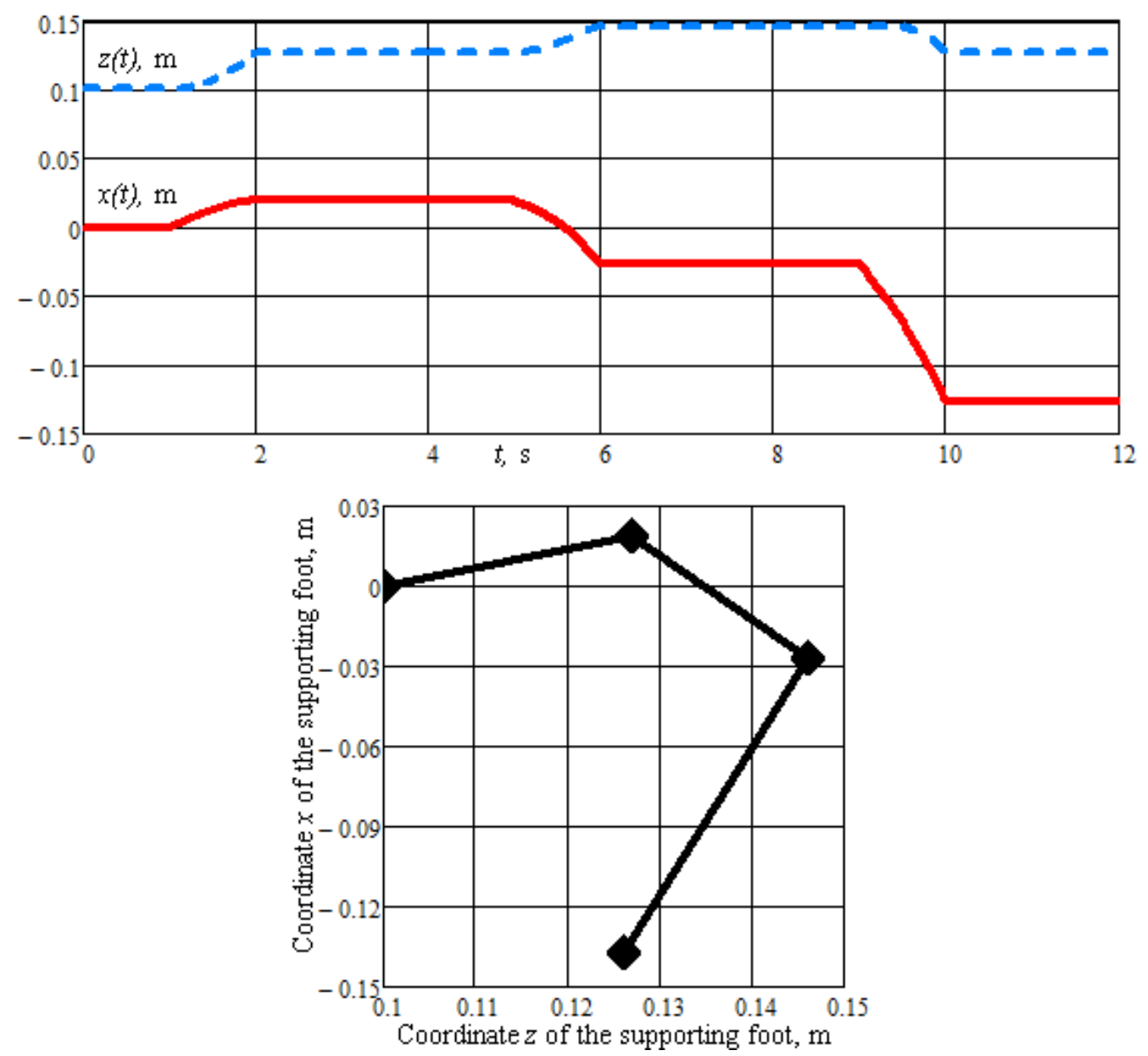

Fig. 6. Time dependencies of changing the coordinates of the robot's supporting foot $(a)$ and the motion trajectory (path) of the supporting foot $(b)$ during the first three steps

\section{Simulation of the Robot Locomotion in SolidWorks Software}

In order to analyze the correctness of the proposed theoretical models and the results of numerical modelling of the robot motion, let us carry out the virtual experiment (computer simulation) using the robot's solid model designed in SolidWorks software (Fig. 7 a). Adopting the same input parameters prescribed above for numerical modelling, the robot motion has been simulated using SolidWorks Motion software. The results of computer simulation are presented in the form of time dependencies of changing the supporting feet coordinates during the robot rectilinear locomotion (Fig. 7 b). 
Vitaliy Korendiy, Oleksandr Kachur, Oleksandr Havrylchenko, Vasyl Lozynskyy
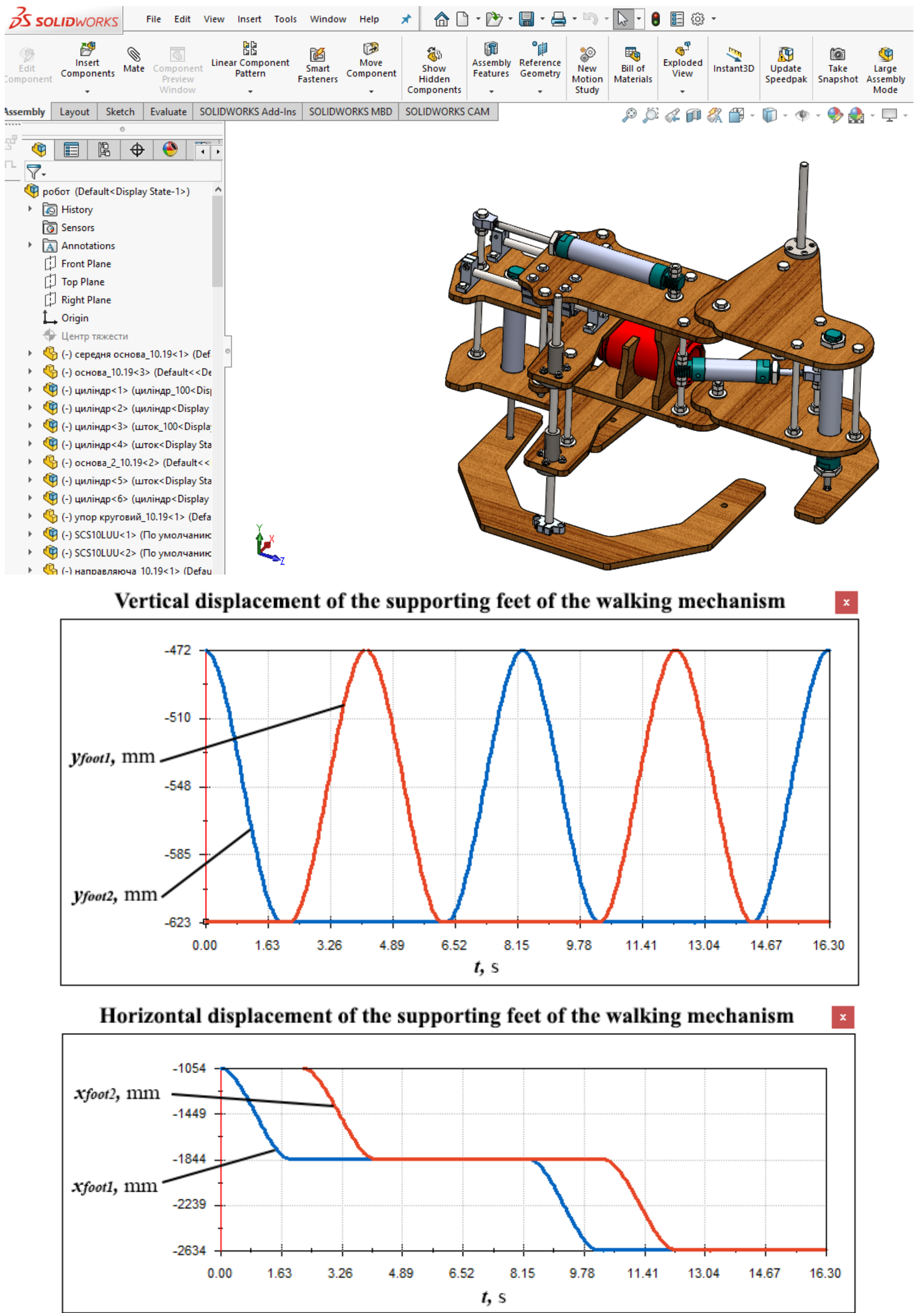

Fig. 7. The robot's solid model designed in SolidWorks software $(a)$ and the simulated time dependencies of changing the coordinates of the robot's supporting feet $(b)$ 


\section{Modelling and Simulation of Pneumatic System Operation of Mobile Robot}

\section{Conclusions}

The paper considers the improved design of the pneumatically-driven orthogonal walking robot equipped with turning mechanism (Fig. 1), analyses its rectilinear and curvilinear locomotion, considers the corresponding timing diagrams of operation of the pneumatic cylinders under the conditions of rectilinear and curvilinear motion (Fig. 2). The simulation model of the robot's pneumatic system was implemented and experimentally tested in FESTO FluidSim Pneumatics software (Fig. 3).

The laws of motion of the main robot's members, which provide its programmable cyclic walking motion, were derived. The numerical modelling of the robot rectilinear and curvilinear motion was performed in MathCAD software (Figs. 5 and 6), and the virtual experiment was conducted in SolidWorks software (Fig. 7). It was established that: 1) the proposed timing diagram of the pneumatic system operation (Fig. 3) is accurately described by the derived equations of the robot's members motion (1); 2) at each moment of time, the supporting feet of at least one platform are in contact with the supporting surface; 3) the supporting feet of different platforms are not at the same walking phase during the whole walking process; 4) the step length during one cycle of the pneumatic system operation ( $4 \mathrm{~s}$ ) equals $0.08 \mathrm{~m}$; 5) the average speed of the robot motion is $0.02 \mathrm{~m} / \mathrm{s}$ or $0.07 \mathrm{~km} / \mathrm{h} ; 5)$ the maximal height of the supporting feet lifting (i.e. the maximal height of the obstacle that can be overcome by the robot) is equal to the maximal extension of the corresponding pneumatic cylinder $-0.08 \mathrm{~m}$.

\section{References}

[1] J. L. Jones, A. M. Flynn, B. A. Seiger, Mobile Robots: Inspiration to Implementation. Boka Raton, Fl: CRC Press, 2019.

[2] J. Angeles, Fundamentals of Robotic Mechanical Systems: Theory, Methods, and Algorithms. Cham, Switzerland: Springer, 2014.

[3] A. J. Kurdila, P. Ben-Tzvi, Dynamics and Control of Robotic Systems. Hoboken, NJ: Wiley, 2019.

[4] X Li, X. Tang, "Position error calibration analysis of a series orthogonal structure robot", Modern Machinery, issue 1, TP242, 2017.

[5] V. Zhoga, V. Skakunov, I. Shamanov, and A. Gavrilov, "Programmable Movement Synthesis for the Mobile Robot with the Orthogonal Walking Drivers", in Advances in Mechanical Engineering. Lecture Notes in Mechanical Engineering. Springer, Cham, 2016, pp. 135-147.

[6] A. E. Gavrilov, V. V. Zhoga, and P. V. Fedchenkov, "Synthesis of optimal program law for movement of a robot with orthogonal walking drives", Journal of Computer and Systems Sciences International, vol. 50, issue 5, pp. 847-857, October 2011.

[7] B. Deepak, M. Bahubalendruni, and B. Biswal, "Development of in-pipe robots for inspection and cleaning tasks", International Journal of Intelligent Unmanned Systems, vol. 4, no. 3, pp. 182-210, 2016.

[8] V. M. Korendiy, O. Yu. Kachur, V. I. Gurey, O. V. Lanets, "Modelyuvannya rukhu mobilnoho robota z pnevmatychnym pryvodom ta ortohonalnym krokuyuchym rushiyem" ["Modelling the motion of mobile robot with pneumatic drive and orthogonal walking mover"], in Proc. of $14^{\text {th }}$ International Symposium of Ukrainian Mechanical Engineers in Lviv, Lviv, Ukraine, May 23-24, 2019, pp. 79-80. [in Ukrainian].

[9] V. Korendiy, R. Zinko, D. Muzychka, "Substantiation of structure and parameters of pneumatic system of mobile robot with orthogonal walking drive", Ukrainian Journal of Mechanical Engineering and Materials Science, vol. 5, no. 1, pp. 61-72, 2019.

[10] V. Korendiy, R. Zinko, Yu. Cherevko, "Structural and kinematic analysis of pantograph-type manipulator with three degrees of freedom", Ukrainian Journal of Mechanical Engineering and Materials Science, vol. 5, no. 2, pp. 68-82, 2019.

[11] V. Korendiy, R. Zinko, V. Lozynskyy, O. Havrylchenko, "Design and operational peculiarities of fourdegree-of-freedom double-legged robot with pneumatic drive and turning mechanism", Ukrainian Journal of Mechanical Engineering and Materials Science, vol. 6, no. 1, pp. 54-71, 2020.

[12] I. L. Krivts, G. V. Krejnin, Pneumatic Actuating Systems for Automatic Equipment: Structure and Design. Boca Raton: CRC Press, 2016.

[13] D. B. Marghitu, Mechanisms and Robots Analysis with MATLAB®. London, UK: Springer-Verlag, 2009.

[14] K. Russell, Q. Shen, R. S. Sodhi, Kinematics and Dynamics of Mechanical Systems Implementation in MATLAB® and SimMechanics®. Boca Raton, Fl: CRC Press, 2019. 